\title{
ON STABILITY AND THE WORST IMPERFECTION SHAPE IN SOLIDS WITH NEARLY SIMULTANEOUS EIGENMODES
}

\author{
Nicolas Triantafyllidis and Ralf Peek \\ College of Engineering, University of Michigan. Ann Arbor, MI 48109, U.S.A.
}

(Receited 22 Octoher 1990; in revised form 15 Nocember 1991)

\begin{abstract}
Of interest here is the influence of imperfections on the stability of elastic systems, discrete or continuous. with nearly simultaneous modes. The majority of such structures show an extreme sensitivity of their first instability load to the shape of these small amplitude but unavoidable imperfections. The determination of the worst such possible shape is thus a very important issue from the design standpoint. Following a general analysis of the stability of arbitrary elastic systems with nearly simultaneous bifurcation eigenmodes in the presence of imperfections. conditions are given for the determination of the worst imperfection shape which minimizes the first local load maximum. For the case of coincident eigenmodes the answer to the worst shape problem is considerably simplified. for it is determined from the equilibrium branch of the perfect structure on which the load drops more rapidly.
\end{abstract}

\section{INTRODUCTION}

Of interest here is the influence of imperfections on the stability of elastic systems (discrete or continuous) with nearly simultancous modes.

The study of bifurcation and stability in structures with simultaneous or nearly simultancous modes is a classical problem in solid mechanics. As such it has received a great deal of attention in engineering literature, especially after Koiter's (1945) pioneering work that put the problem on a sound mathematical basis. In addition to its theoretical interest. and accompanying inherent difliculties. the problem is also of great practical importance. There is a variety of enginecring applications that exhibit multiple bifurcation points at their first buckling loads. Perhaps the best known such examples are the thin walled shell type structures such as thin walled beams, cylinders, cylindrical panels, spheres and spherical caps. The thinner such structures are, the greater the number of buckling modes that appear almost simultaneously near the lowest critical load. Another set of applications contains stiffened structures, such as rib stiffened plates and cylindrical panels as well as large frame type space structures in which a fundamental unit cell can be identified. Due to their particular geometry, both a global and a local buckling mode can occur at, or nearly at, the same load level. The global modes, which have a characteristic wavelength on the order of the dimensions of the structure interact with the local modes whose characteristic wavelength is of the order of the unit cell size. Finally, an additional interesting application pertains to structures that have been optimized with respect to their lowest buckling loads. The optimization procedure leads to overlapping with the next higher buckling load. The higher the number of available design parameters, the more simultaneous modes appear in the optimized structure. For these structures, the first instability point (i.e. the first bifurcation point or local load maximum) can be extremely sensitive to the shape of imperfections. The determination of the worst such possible shape is thus a very important issue from the design standpoint and is the focus of this paper.

Only a brief discussion of the literature pertaining to instability behavior and the first maximum load of imperfect systems with simultaneous (or nearly simultaneous) eigenmodes will be presented. Attention will be further restricted to general formulations of the problem rather than the solution of particular applications. The approach of interest here is based on the decomposition of the space containing all the admissible displacement fields into two parts : one part contains all the $m$ eigenvectors of the stability operator (where $m$ is an integer) and is called the null space of the operator, while the other part is the orthogonal complement to the null space. This procedure considerably simplifies the required work 
since it reduces the study of an infinite dimensional problem to the study of a finite $m$ dimensional one. In mathematics this decomposition is called the Lyapunov-Schmidt decomposition. It has been introduced to the engineering literature in Koiter's (1945) work on elastic stability.

The Lyapunov-Schmidt-Koiter ( $\mathrm{L}-\mathrm{S}-\mathrm{K})$ decomposition is usually combined with an asymptotic analysis of the equilibrium equations near the critical point. In this approach a solution is first obtained for the part of the equilibrium equation that pertains to the orthogonal complement of the null space. These results are then introduced into the equilibrium equation along the null space. The form of the asymptotic expansion of the equilibrium equations as well as the stability results for the perfect or imperfect structures are obtained in a simple and straightforward fashion from the analysis. A very good description of this method can be found in Potier-Ferry (1979) as well as in the book of looss and Joseph (1980). A finite degree of freedom version for the study of equilibrium using this type of $\mathrm{L}-\mathrm{S}-\mathrm{K}$ decomposition is also given by Thompson and Hunt (1973).

The main concern in the engineering literature is for the equilibrium solution of the imperfect structures in question [see for example the review article by Budiansky (1974)]. For a perfect structure, the asymptotic analysis for the minimum eigenvalue of the stability operator along the bifurcated equilibrium branch is given by Budiansky (1974) for the simple eigenmode and by looss and Joseph (1980) for the multiple eigenmode asymmetric case. An asymptotic analysis for the stability of the equilibrium solutions in imperfect structures with nearly simultaneous modes by means of calculating the lowest eigenvalue of the stability operator has not been presented up to date, to the best of our knowledge. The above mentioned asymptotic stability analysis is subsequently employed in the formulation of the worst imperfection shape problem. for which the necessary conditions are derived.

The outline of the presentation is as follows: Section 2 presents the asymptotic equilibrium and stability analysis for the perfect structure with simultaneous eigenmodes using the above described L-S.K approach. Although the results of this section are known [e.g. looss and Joseph (1980)], their presentation is indispensable for the development of the subject. The symmetric multiple bifurcation stability analysis, which is also of practical interest (its applications include plates, frames etc.) has only been presented so far under the somewhat more restrictive conditions of linear prebuckling [see Byskov and Hutchinson (1977) who are mainly interested in the equilibrium of these structures]. Hence the general presentation of the symmetric case is adding to the interest of this section.

In Section 3, the asymptotic L-S-K analysis is generalized to include the imperfections. One difference in the results obtained here from what is usually presented in the existing literature, is the distinction of two different control parameters: one, denoted by $\varepsilon$, is related to the geometrical (in a more general sense) shape of the structure and the other, denoted by $\zeta$, characterizes the critical load spacing of the interacting eigenmodes. This last parameter, termed here the mode separation parameter, is also called the splitting parameter by Thompson and Hunt (1973) and is an average measure of the difference in the critical loads of the nearly simultaneous-and thus interacting-eigenmodes. The first instability load is found for both asymmetric and symmetric bifurcations where $\varepsilon, \zeta \neq 0$.

Section 4 investigates the issue of the worst imperfection shape. Necessary conditions are found which maximize the difference between the critical load of the perfect structure and the first instability load of the imperfect one over all the possible shapes of the geometric imperfection. For the particular case when the mode separation parameter is negligible compared to the geometric imperfection amplitude $(\varepsilon \neq 0, \zeta=0)$ and when a particular choice of inner product is used for the admissible displacement space, the above mentioned conditions simplify considerably. It is shown that the worst shape always corresponds to a maximum load and that it can be found by determining the bifurcated branch with the steepest load drop in the perfect structure with the simultaneous modes. This particular result was proven using a different approach by Koiter (1976) [see also Ho (1974)]. For the general case $\varepsilon, \zeta \neq 0$ the worst imperfection shape problem requires the solution of a more complicated problem which also depends on the adopted parameterization of the 
equilibrium equations. An asymptotic lower bound for the maximum load drop is also provided in this case.

The novel results in this paper are essentially derived in Section 4. The material presented in the previous sections (and which is indispensable for the analysis in Section 4) although known. is scattered in various places in the engineering and mathematics literature and treated under different notations and often using unnecessary restrictions. We believe that the presentation of this material using a unified and concise approach under the least restrictive conditions possible brought together in one reference also serves a useful purpose.

The presentation is concluded by a discussion of the general results obtained in this work.

\section{BIFURCATION AND STABILITY ANALYSIS OF THE PERFECT STRUCTURE}

The essential features of the problem are embodied in the perfect structure with the simultaneous eigenmodes at bifurcation. The analysis of the imperfect structure with the nearly simultaneous eigenmodes requires finding the multiple equilibrium paths of the perfect structure and the determination of their stability. Consequently, this presentation will start with the study of the bifurcated equilibrium paths of the perfect structure.

In the present work, only nonlinear elastic structures are to be studied. To this end, consider a structure whose potential energy is given by $\mathscr{L}(u, \lambda)$. Here $u$ denotes the displacements of the structure from the stress free reference configuration and belongs to a vector space of admissible functions denoted by $U$. In the applications of interest, an inner product can be defined on $U$, and the inner product of $u_{1}, u_{2} \in U$ is denoted by $\left(u_{1}, u_{2}\right)$. The corresponding norm is $\|u\| \equiv(u, u)^{1 / 2}$. Moreover the scalar $\lambda$ denotes the load parameter of the system and increases monotonically away from zero during the loading process. For $\lambda=0$, the equilibrium solution is the stress free configuration with displacement $u=0$. For values of the load parameter $0 \leqslant \lambda<\lambda_{s}$, the equilibrium solution of the perfect structure, denoted by $\ddot{u}(\lambda)$ and called principal solution, is unique and stable. More specifically, for any $\lambda \in\left[0, \lambda_{\mathrm{s}}\right)$ there exists a real number $\alpha(\lambda)>0$ for which the following relations are satisfied : $†$

$$
\mathscr{E}_{. u}(\stackrel{0}{u}(\lambda), \lambda) \delta u=0 ;\left(\mathscr{S}_{. u u}(\stackrel{0}{u}(\lambda), \lambda) \delta u\right) \delta u>\alpha\|\delta u\|^{2}, \quad \forall \delta u \in U
$$

In the above equation, the first relation indicates that $\stackrel{0}{u}(\lambda)$ is an equilibrium solution for the perfect structure with potential energy 8 . The second relation ensures its stability, over the considered range of the load parameter $\lambda$, since it implies a strong local minimum of the potential energy.

As the load parameter increases away from zero, it reaches a value called the critical load and is denoted by $\lambda_{c}$, for which the principal solution loses its stability, i.e. the principal solution is no longer a strong local minimum of the potential energy. Consequently the minimum eigenvalue of the stability operator $\delta_{\text {.um }}$ evaluated on the principal solution and at the critical load should vanish. At criticality, the corresponding stability operator has $m$ simultaneous eigenmodes denoted by $\dot{u}$. In mathematical terms :

$$
\delta_{. \mu i}\left(u\left(\lambda_{c}\right), \lambda_{c}\right) \dot{u}=0, \quad\left(\mathscr{E}_{., u u}\left(\stackrel{0}{u}\left(\lambda_{c}\right), \lambda_{c}\right) \dot{u}\right) \delta u=0 ;(u, u)=\delta_{\imath,}, \quad i, j=1, \ldots, m .
$$

The second relation in (2) expresses the fact that $i$ is one of the $m$ simultaneous eigenmodes of the stability operator of the perfect structure at criticality while the first relation in the same equation indicates that the critical point is a true $m$-fold birfurcation point and not an extremum load point. In almost all the applications of interest in structural and solid mechanics, the number of the simultaneous eigenmodes of the stability operator 
at criticality is finite. The third condition in (2) (where $j_{i,}=1$ if $i=j$ and $\delta_{i,}=0$ otherwise) ensures that the eigenmodes form an orthonormal set. This property will facilitate some of the subsequent calculations. Further simplification can be achieved by choosing a specific inner product. as will be described later.

The main tool of the analysis is what is called in the mathematics literature the LyapunovSchmidt decomposition of the displacement field $u$. According to this method, $u-u(\lambda)$ can be decomposed in two parts: one which lies in the null space of the stability operator at criticality,

$$
1 \equiv\left\{u \in l \mid u=\sum_{i=1}^{m} \sum_{i}^{i} u . \quad \zeta_{i} \in \mathbb{R} ;\right.
$$

and one which lies in its orthogonal complement with respect to $U$.

$$
1^{-1} \equiv\left\{r \in U \mid(c, u)=0, \quad i=1, \ldots, m_{\}}\right\}
$$

More specifically:

$$
u=u(j)+\sum_{i=1}^{m} \xi_{i} u+\imath ; \xi_{1} \in \boldsymbol{M} . \quad \imath \in \cdots
$$

In addition, it follows from (1), (2) and the continuity, with respect to the load parameter, of the stability operator evaluated on the principal equilibrium path, that at criticality the stability operator must be positive semidefinite on $U$. This implies its strict positive definiteness on $1^{\prime 4}$. Thus there exists a real number $\alpha>0$ [unrelated in general to the one defined in (1)] for which:

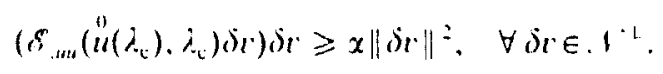

The sought displacement $"$ is thus replaced by an equivalent set of unknowns $\left(l, \zeta_{1}\right)$ and the solution to the equilibrium equation $\mathscr{f}_{u} \delta u=0$ proceeds in two steps: first $r$ is determined as a function of $\Delta \lambda\left(\Delta \lambda \equiv \lambda-\lambda_{e}\right)$ and $\xi$, from the equilibrium equation in $1^{1}$. Then the resulting $r$ is used in the remaining equilibrium equation on. 1 . This provides the relation between $\Delta x$ and $\xi_{\text {. }}$. The equilibrium equations that have to be solved are:

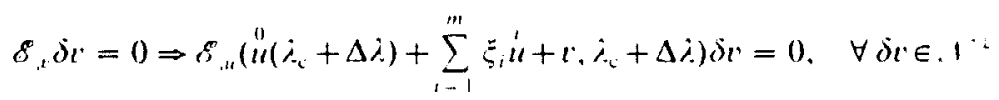

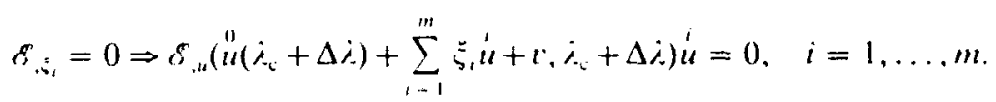

From (4) it follows that (5), has a unique and adequately smooth solution $c\left(\xi_{1}, \Delta j\right)$, at least in the neighborhood of the critical point $\left.\left(u(i), j_{i}\right), j_{c}\right)$. The Taylor series expansion of this solution is:

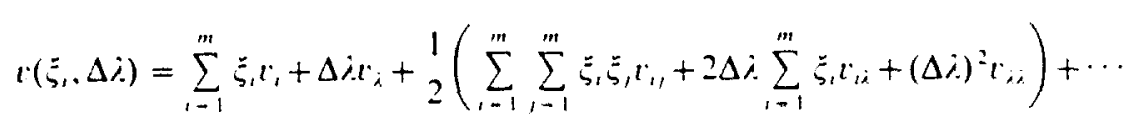

Upon substitution of (6) into (5), and subsequent evaluation of its Taylor series expansion about $\left(\xi_{1}, \Delta \lambda\right)=0$ one obtains the following results: the $O(1)$ term of the expansion gives $+\sigma^{\mathrm{c}} \delta \mathrm{s}=0$ which is automatically satisfied in view of the equilibrium

\footnotetext{
tFrom here on the superseript ( $f^{\prime}$ or subscript () denotes evaluation of the quantity in question at the critical point. $\left(a\left(i_{x}\right), j_{x}\right)$.
} 
equation (1). The $O\left(\xi_{i}\right)$ terms yield, in conjunction with (2), the result : $\left(\mathscr{E}_{.44}^{c} v_{i}\right) \delta v=0$ which in view of ( 4$)$ implies:

$$
v_{i}=0
$$

By taking $\bar{\zeta}_{:}=0$ into $(5)_{1}$. comparing the result to the equilibrium condition on the fundamental solution ( 1$)$, and invoking the uniqueness of the solution to $(5)$, for $i\left(\xi_{i}, \Delta \lambda\right)$, it is readily seen that $c(0, \Delta \dot{i})=0$. This implies:

$$
v_{i}=v_{i i}=\cdots=0
$$

This result could also have been obtained from the $O(\Delta \lambda)^{n}$ terms in the expansion of (5),

One can similarly continue with the quadratic order terms in the expansion of the equilibrium equation (5), to find:

$$
\begin{aligned}
& O\left(\xi_{i}, \xi_{i}\right) \operatorname{term}:\left(\mathscr{E}_{. u u}^{\mathrm{c}} v_{i j}+\left(\mathscr{E}_{\text {.uuu }}^{\mathrm{c}} u\right)^{i} u\right) \delta v=0 \\
& O\left(\xi_{i} . \Delta \lambda\right) \operatorname{term}:\left(\mathscr{E}_{\text {.uu }}^{\mathrm{c}} u_{i i}+\left(\mathrm{d} \mathscr{E}_{. \text {uu }} / \mathrm{d} \lambda\right)_{\mathrm{v}} u\right) \delta v=0
\end{aligned}
$$

The above equations have unique solutions $v_{i}, v_{i d}$ in view of (4). In a similar way one can find the higher order terms in the expansion of $c\left(\xi_{i}, \Delta \lambda\right)$ and hence determine the solution to (5), uniquely. Upon substitution of the thus found $v\left(\xi_{i} . \Delta \lambda\right)$ into the remaining equilibrium equation (5).. and after using (2). (7). (8) one obtains the following $m$ equations between $\Delta \lambda$ and $\xi_{;}$:

$$
\begin{aligned}
& 2\left[\sum_{-1}^{m} \sum_{k=1}^{m} \xi_{1} \xi_{k} \delta_{1, k}+2 \Delta i \sum_{i=1}^{m} \xi_{1} \delta_{1, \lambda}\right]+\frac{1}{6}\left[\sum_{i=1}^{m} \sum_{k=1}^{m} \sum_{1=1}^{m} \xi_{i} \xi_{k} \xi_{1} \delta_{1, k l}+\cdots\right]+\cdots=0
\end{aligned}
$$

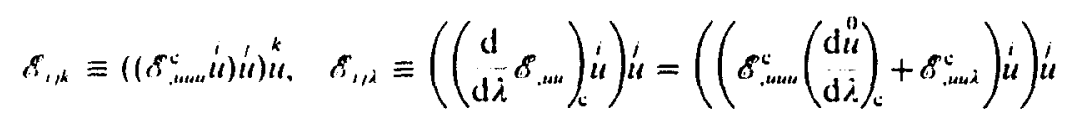

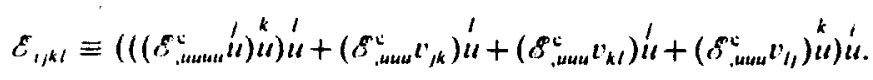

As expected. an obvious solution to $(10)$ is the principal equilibrium branch for which $\xi_{t}=0$ and $\Delta \lambda \neq 0$ since from (3) and (6), one obtains for this case $u=u(\lambda)$. The determination of the remaining equilibrium paths through the critical point, i.e. the determination

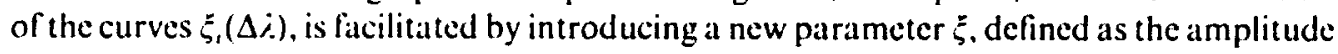
of the projection of $\|-\|$ on the null space $l$ of $\xi_{u u}$. Needless to say, the above parametrization is not the only possible one. However, it turns out to be the most convenient one for the ensuing calculations. For a neighborhood of the critical point, assuming an adequately smooth dependence of $\xi_{\text {. }} \Delta \lambda \dot{\lambda}$ on $\zeta$ one has:

$$
\begin{aligned}
\zeta_{1}(\xi) & =x_{1}^{1} \xi+x_{i}^{2} \frac{\xi^{2}}{2}+\cdots \\
\Delta \lambda(\xi) & =\lambda_{1} \xi+i_{2} \frac{\xi^{2}}{2}+\cdots
\end{aligned}
$$

Two cases are to be distinguished at this point: first the asymmetric case for which $\delta_{i j k} \neq 0$ at least for one triplet of indices $(i, j, k)$. By inserting (11) into (10) and collecting the terms of the like order in $\bar{\zeta}$, one obtains from the lowest order nontrivial term in this expansion (the $O\left(\xi^{2}\right)$ term) : 


$$
\sum_{j=1}^{m} \sum_{k=1}^{m} x_{i}^{\prime} x_{k}^{1} \mathscr{E}_{i j k}+2 i_{1} \sum_{i=1}^{m} x_{j}^{i} E_{i, j}=0 . \quad \sum_{i=1}^{m}\left(x_{i}^{\prime}\right)^{2}=1
$$

The above algebraic system of $m+1$ equations for the $m+1$ unknowns $x_{1}, i_{1}$ has at most $2^{m}-1$ pairs of real solutions $\left(x_{1}^{1}, \lambda_{1}\right)$ and $\left(-x_{1}^{1},-\lambda_{1}\right)$. with each pair corresponding to a bifurcated equilibrium path through the critical point. Each one of these equilibrium paths can be constructed by computing its Taylor series expansion as indicated in (11). Higher order terms in the expansion of (10) show that the series can be continued to any desired order if :

$$
\operatorname{Det}\left[B_{1,}\right] \neq 0, \quad B_{1,} \equiv \sum_{k=1}^{m} x_{k}^{1} \sigma_{1 / k}+i_{1} \varepsilon_{1, i}
$$

The second case to be investigated will be the symmetric case for which $\varepsilon_{i, k}=0$ for all triplets of indices $(i, j, k)$. In this case (12) implies that $\lambda_{1}=0$. By inserting (11) into (10) and collecting the terms of the like order in $\zeta$. one obtains from the lowest order nontrivial term in this expansion (the $O\left(\xi^{3}\right)$ term) :

$$
\sum_{i=1}^{m} \sum_{k=1}^{m} \sum_{i=1}^{m} x_{i}^{\prime} x_{k}^{\prime} x_{i}^{\prime} \delta_{i, k l}+3 \lambda_{2} \sum_{i=1}^{m} x_{i}^{\prime} \delta_{i, a}=0, \quad \sum_{i=1}^{m}\left(x_{r}^{\prime}\right)^{2}=1
$$

This algebraic system of $m+1$ equations for the $m+1$ unknowns $x_{1}^{1}, \lambda_{2}$ has at most $\left(3^{\prime \prime}-1\right) / 2$ pairs of real solutions $\left(x_{i}^{\prime}, \lambda_{2}\right)$ and $\left(-x_{1}^{\prime}, \lambda_{2}\right)$. each corresponding to a bifurcated equilibrium path through the critical point. Each one of these equilibrium naths can be constructed by computing its Taylor series expansion as indicated in (11). The continuation of the expansion (10) to terms of $O\left(\xi^{-1}\right)$ and higher for each particular branch is assured when:

$$
\operatorname{Det}\left[B_{i j}\right] \neq 0, \quad B_{1,} \equiv \sum_{k=1}^{m} \sum_{i=1}^{m} x_{k}^{1} x_{l}^{\prime} \sigma_{i, k]}+\lambda_{2} \kappa_{i, j}
$$

To complete the study of the above found bifurcated equilibrium branches, one has to investigate their stability. The general dynamic stability criterion applied to structures with a potential energy can be shown to be essentially equivalent to the sign of the minimum cigenvalue $\beta^{\text {mun }}$ of the stability operator $\mathscr{E}_{. u 4}(u, \lambda)$ evaluated on the equilibrium path whose stability is under investigation. For $\beta^{\text {min }}>0$, the potential energy has a strict local minimum and the corresponding equilibrium solution can be shown to be stable (for the continuum case, some additional minor technical conditions on $E(u, \lambda)$ and $\|u\|$ have to be verified). For $\beta^{\prime \prime \prime n}<0$. the equilibrium solution does not correspond to a local minimum of the potential energy and hence the solution can be shown to be unstable. A word of caution is in line at this point. The sign of $\beta^{\text {min }}$ (i.e. whether $\beta^{\text {man }}$ is positive, zero or negative) is independent of the choice of norm for the catse of finite degree of freedom systems. For infinite systems, the sign of $\beta^{\text {min }}$ depends on the adopted norm. For most of the practical applications of interest, there are fortunately physically motivated choices for the norm in the admissible displacement space $U$ which give stability results in agreement with physical intuition. For further discussion on this point the interested reader is referred to Koiter (1965), Knops and Wilkes (1973) and Simpson and Spector (1987).

First the stability of the principal branch is to be investigated. To this end, assume

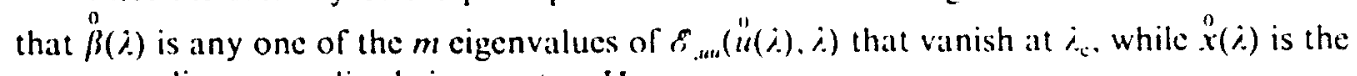
corresponding normalized eigenvector. Hence:

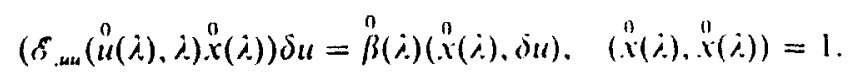

Evaluating (16) at the critical load one has in view of (2): : 


$$
\stackrel{0}{x}_{c}=\sum_{i=1}^{m} \ddot{x}_{i}^{0} u
$$

Assuming that $\stackrel{0}{\beta}(\dot{j})$ and $\stackrel{0}{x}(\dot{\lambda})$ are smooth functions of their argument, $(16)$ can be differentiated with respect to $\lambda$. Evaluating the result at the critical point, and recalling that $\stackrel{p}{\beta}\left(i_{\mathrm{v}}\right)=0$ one obtains:

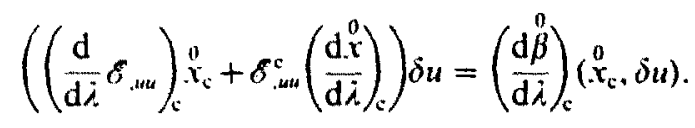

Taking $\delta u=i$ and recalling $(2)_{2},(16)_{2}$, eqn $(18)$ yields :

$$
\sum_{i=1}^{m} E_{i j i} \ddot{\chi}_{i}=\left(\frac{\mathrm{d} \beta}{\mathrm{d} \lambda}\right)_{i} \ddot{\chi}_{i}
$$

Since $\stackrel{b}{\beta}(\lambda)$ is positive for $\lambda<\lambda_{\mathrm{c}}$ and vanishes at $\lambda_{\mathrm{c}}$, it follows from continuity that the derivative of this eigenvalue evaluated at criticality. $(d / \beta / d \lambda)_{i}$, cannot be positive. Assuming that this quantity is strictly negative, i.c. $(\mathrm{d} B / \mathrm{d} \lambda)_{\mathrm{c}}<0$. leads to the conclusion that $E_{i j \dot{\alpha}}$ must he a negative definite matrix. This condition is satisfied in the majority of applications of interese here.

For each bifurcated equilibrium branch trough the critical point, its stability depends on the sign of the minimum eigenvalue $\beta^{\text {man }}(\xi)$ of the corresponding stability operator $\delta$...u. In analogy to (16), the definitions for each one of the $m$ lowest eigenvalues $\beta(\xi)$ and the corresponding normalized eigenvectors $x(\bar{\zeta})$ are:

$$
\left(\delta_{\text {aut }}\left(u\left(\lambda_{\mathrm{c}}+\Delta \lambda\right)+\sum_{i=1}^{m} \xi_{i} u+r\left(\xi_{i}, \Delta \lambda\right), \lambda_{\mathrm{c}}+\Delta \lambda\right) x\right) \delta u=\beta(x, \delta u), \quad(x, x)=1
$$

In the above definition $\xi_{1}, \Delta \lambda, \beta, x$ are functions of the parameter $\xi$. Recall that for every bifurcated equilibrium path the $m$ lowest eigenvalues $\beta(\xi)$ of the corresponding stability operator have to vanish at $\xi=0$. In addition, for each bifurcated equilibrium path, $\beta(\xi)$ and $x(\xi)$ are assumed to be smooth functions of their argument with the following Taylor series expansions:

$$
\begin{aligned}
& \beta(\xi)=\beta_{1} \xi+\beta_{2} \frac{\xi^{2}}{2}+\cdots \\
& x(\xi)=x_{0}+x_{1} \xi+x_{2} \frac{\xi^{2}}{2}+\cdots .
\end{aligned}
$$

By introducing (21) into (20) and recalling (2), (6)-(8), (11), one obtains by expanding about $\xi=0$ and collecting the terms of the like order in $\bar{\xi}$ the following results: the $O(1)$ term yiclds:

$$
x_{n}=\sum_{i=1}^{m} x_{1}^{i} u
$$

Continuing with the $O(\xi)$ term in the expansion of $(20)$ one has: 


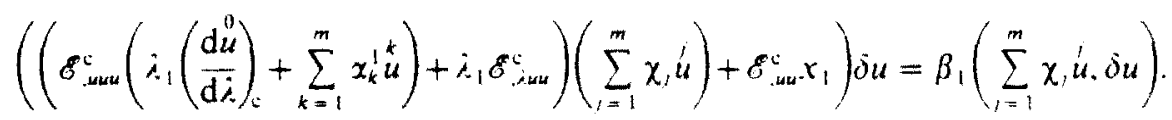

Taking $\dot{\partial} u=\dot{u}$ and recalling (2), the above equation yields:

$$
\sum_{i=1}^{m} B_{i} \chi_{i}=\beta_{1} \chi_{i}
$$

where $B_{i j}$ is given by (13). For the asymmetric bifurcation case, this matrix is nonsingular. which ensures that all its eigenvalues $\beta_{1}$ are nonzero (as well as real in view of the symmetry

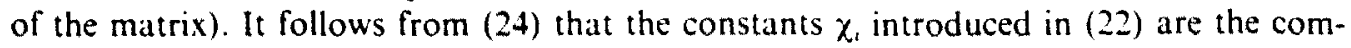
ponents of the eigenvector of $B_{i}$ corresponding to the eigenvalue $\beta_{1}$. Hence the minimum eigenvalue $\beta^{\min }(\xi)$ of $\mathscr{E}_{\text {.ut }}$ for the bifurcated equilibrium path in question is :

$$
\beta^{\min }(\xi)=\left\{\begin{array}{lll}
\xi \beta_{1}^{\operatorname{mix}}+O\left(\xi^{2}\right) & \text { if } \xi<0 \\
\xi \beta_{1}^{\min }+O\left(\xi^{2}\right) & \text { if } \xi>0 .
\end{array}\right.
$$

Consequently. if for a certain bifurcated branch the maximum and minimum eigenvalues of $B_{i}$, respectively $\beta_{1}^{\max }$ and $\beta_{1}^{m+n}$ are of the same sign, then the bifurcated branch in question changes stability as it crosses the critical point, while if the two extremal eigenvalues are of opposite sign the bifurcated branch in question is always unstable.

For the symmetric bifurcation case, since $\delta_{i, k}=\lambda_{1}=0, B_{t}=0$ in (24) which implies that $\beta_{1}=0$. Consequently, one has to consider the $O\left(\xi^{2}\right)$ term in the expansion of $(20)$. which with the holp of $(2),(6)(8),(11),(21)$ yiclds:

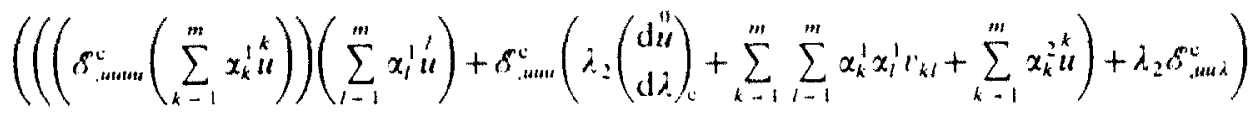

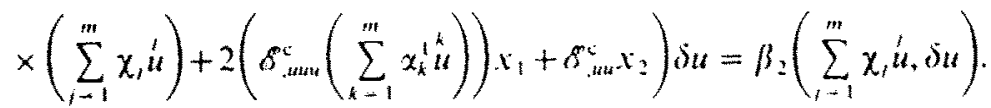

From (23) and since $\lambda_{1}=\beta_{1}=0$ one obtains with the help of (9), that:

$$
x_{1}=\sum_{i=1}^{m} \sum_{i=1}^{m} x_{i} x_{i}^{1} v_{i j}+\bar{x}_{1}
$$

with $\tilde{x}_{1} \in \psi$. By subsequently taking $\delta u=\dot{\alpha}$ and using (27) into (26) one obtains:

$$
\sum_{i=1}^{m} B_{i,} \chi_{i}=\beta_{2} \chi_{i}
$$

where $B_{i}$ is now given by (15). For the symmetric bifurcation case, this matrix is nonsingular, which ensures that all its eigenvalues $\beta_{2}$ are nonzero (as well as real in view of the symmetry of the matrix). It follows from (28) that the constants $\chi_{\text {i }}$ introduced in (22) are the components of the eigenvector of $B_{i}$, corresponding to the eigenvalue $\beta_{2}$. Hence the minimum eigenvalue $\beta^{\min }(\xi)$ of $\delta_{\text {.um }}$ for the corresponding bifurcated equilibrium path in question is:

$$
\beta^{\min }(\xi)=\sum_{2}^{\xi^{2}} \beta_{2}^{\min }+O\left(\xi^{3}\right)
$$

Consequently if the minimum eigenvalue $\beta_{z}^{\text {mun }}$ of $B_{i j}$ is positive, the bifurcation branch in question is stable. Any negative eigenvalues render it unstable. 


\section{EQUILIBRIUM AND STABILITY OF THE IMPERFECT STRUCTURE}

Having analysed the bifurcation and stability behavior of a perfect structure with a multiple bifurcation point, attention is turned to the effect of imperfections. The term "imperfections" is employed in a very broad engineering sense here, as opposed to the more rigorous mathematical term "control parameters" of singularity theory [see Golubitsky and Schaeffer (1985)]. Guided by the vast majority of applications in solid and structural mechanics, two types of such imperfections (or control parameters) will be distinguished: the first is a geometric imperfection. denoted by $w$, where $w$ is a function which characterizes the departure of the unstressed configuration of the structure from its perfect shape. More generally, a geometric imperfection can be due to the eccentricity of the applied loads. thickness variations, errors in the boundary conditions. residual stresses etc. The second is the mode separation parameter [also referred to as "mode splitting" parameter by Thompson and Hunt (1973)]. denoted by $\zeta$. which quantifies the average distance of the nearly simultaneous critical loads. Note that mode separation can occur in perfect structures. such as rectangular plates under in-plane loading. axially loaded cylinders or externally pressurized spheres that exhibit no geometric imperfections of any kind. Although one can find examples of strongly interacting modes with critical loads that are far apart [see for example Healey (1988)] attention is focused here on the interaction of almost simultaneous modes, i.e. eigenmodes with corresponding critical loads that are close to each other.

The potential energy of an imperfect structure with nearly simultaneous eigenmodes is given by $\bar{f}\left(u, \lambda, \zeta, w^{\circ}\right)$. Here " again denotes the displacement of the structure from some reference configuration and $n$ denotes the geometric imperfection of the structure. It will be assumed that $u \in U$ while $n \in W$ where $W$ is a vector space of admissible geometric imperfections. It is convenient to distinguish between the imperfection amplitude $\varepsilon \equiv\|w\|$ and the imperfection shape $\vec{w} \equiv w^{\prime} /\left\|w^{\prime}\right\|$. Moreover the scilar $\lambda \geqslant 0$ denotes once more the load parameter of the system and the scalar $\zeta \in \mathbb{N}$ is the mode separation parameter. $A$ structure with nearly simultaneous eigenmodes but with no geometric imperfection has a potentiat energy $\delta(u, \lambda, \zeta) \equiv \bar{\delta}(u, \lambda, \zeta, 0)$. The potential energy of the perfect structure with the simultaneous cigenmodes obviously satisfies $\mathscr{\delta}(u, \lambda)=\mathscr{\delta}(u, \lambda, 0)$. The relations between the energies of these different structures are thus summarized:

$$
\delta(u, \lambda)=\hat{\delta}(u, \lambda, 0), \quad \dot{\delta}(u, \lambda, \zeta)=\vec{E}(u, \lambda, \zeta, 0) ; \quad u \in U, \quad u \in W, \lambda \geqslant 0, \quad \zeta \in \Downarrow
$$

The perfect structure with the nearly simultaneous eigenmodes has a principal solution $\ddot{u}(j, \zeta)$ which in view of $(1)$, and $(30)$, satisfies:

$$
\hat{\delta}_{. u}(\hat{u}(\lambda, \zeta), \lambda, \zeta) \delta u=0, \quad \stackrel{0}{u}(\dot{\lambda})=\stackrel{0}{u}(\lambda, 0)
$$

Moreover, for the perfect structure with the nearly simultaneous modes, one has $m$ distinct critical values $\dot{\hat{h}}(\zeta)$ of the load parameter at the neighborhood of $\lambda_{\mathrm{w}}$. Each such critical value corresponds to a singular point of the stability operator $\left.\hat{\delta}_{\text {..un }}(\dot{0}(\lambda), \zeta), \lambda, \zeta\right)$ with eigenvector $\dot{u}(\zeta)$. All these critical values are close to $\lambda_{\mathrm{c}}$ in the sense that $\dot{\hat{\lambda}}(\zeta)-\lambda_{\mathrm{c}}=O(\zeta)$. More specifically for $i=1, \ldots, m$ :

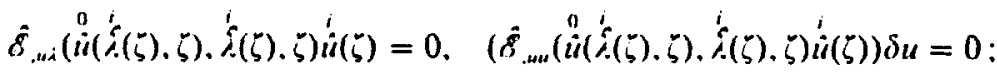

$$
\begin{aligned}
& \dot{f}(0)=i, \quad \dot{u}(0)=\dot{u} .
\end{aligned}
$$

From the above properties of $\dot{\delta}(u, \lambda, \zeta)$ one can deduce the following useful relations: by evaluating the derivative with respect to $\zeta$ of the equilibrium equation (31) at $\zeta=0$ and making use of (30) one has: 


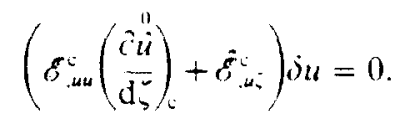

Upon taking $\delta u=u$ and recalling (2), the above equation yields:

$$
\hat{E}_{u:}: \dot{u}=0
$$

By evaluating the derivative with respect to 5 of $(32)$ at $\square=0$ and recalling once again (30) one obtains :

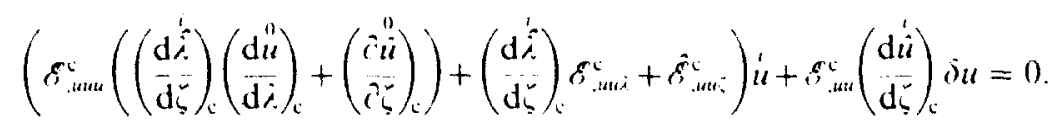

Upon taking $\delta u=i$ and recalling (2) as well as the definition of $\delta_{1, i}$ given in (10) the above equation yields:

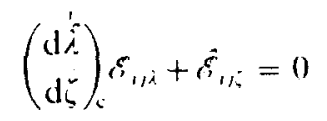

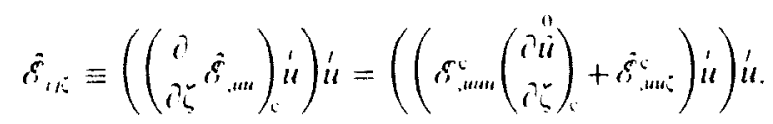

As will be discussed in the next section, there exist appropriate choices of the inner product in $u$ such that the $m \times m$ matrix $\delta_{1, i}=-\delta_{i,}$. Hence from (36) one can conclude that for this convenient inner product choice, $\dot{\delta}_{1,5}$ is also diagonal.

The Lyapunov Schmidt decomposition of the displacement ficld $u$ introduced in (3),

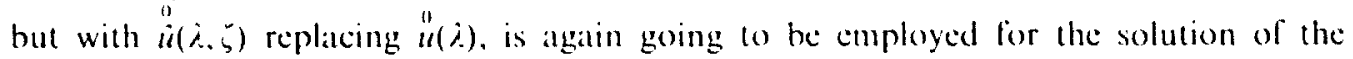
equilibrium equations of the imperfect structure. The unknown displacement $u$ is thus replaced by an equivalent set $\left(l, \xi_{1}\right)$ and the solution to the equilibrium equation $\vec{\delta}_{u} \delta_{a}=0$ proceeds in two steps: first $c$ is determined as a function of $\zeta_{1}, \Delta \lambda, \zeta, \varepsilon$ from the equilibrium equation in.$^{1}$. Then the resulting $v$ is used in the remaining equilibrium equation on. $r$ thus providing the relation between $\xi_{,}, \Delta \lambda, \zeta$ and $i$. The equilibrium equations that have to be solved are:

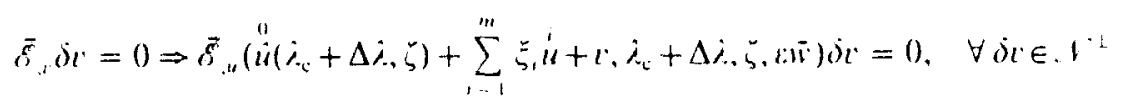

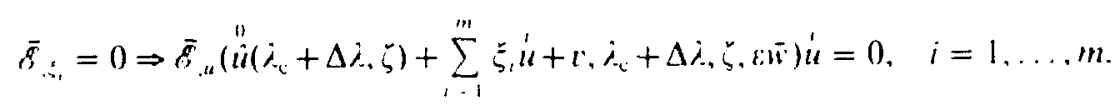

From (4) and (30), it follows that (37), has a unique and adequately smooth solution $i\left(\xi_{1}, \Delta \xi_{,}, 8\right)$, at least in the neighborhood of criticality, whose Taylor series expansion is of the form:

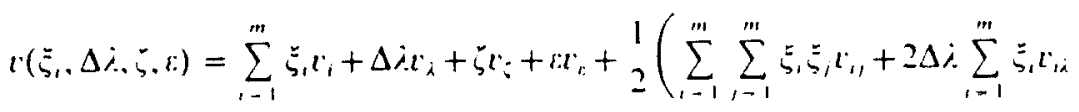

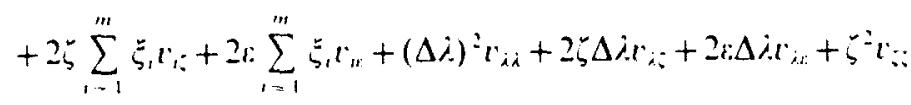

$$
\begin{aligned}
& \left.+2 \zeta \varepsilon v_{v s}+\varepsilon^{2} v_{c \alpha}\right)+\cdots
\end{aligned}
$$

Upon substitution of (38) into (37), and a subsequent Taylor series expansion about 
$\left(\xi_{i}, \Delta \zeta_{,}, \varepsilon\right)=0$ one obtains by collecting the terms of the like orders in these variables the following results: since from $(30), \mathscr{E}(u, j, 0,0)=\mathscr{E}(u, \lambda)$ all the terms in the expansion of (37), of $O\left(\zeta^{p}(\Delta \lambda)^{4}\right)$, i.e. the terms which do not contain any powers of $\varepsilon$ or $\zeta$, coincide with those found in the previous section for the perfect structure [see equations (7)-(9)]. By taking $\xi_{1}=\varepsilon=0$ in (37), comparing the result to the equilibrium equation in (31) and invoking the uniqueness of the solution to $(37)$, for $c\left(\zeta_{i}, \Delta i, \zeta, \varepsilon\right)$, it is readily seen that $v(0, \Delta i . \zeta, 0)=0$. This implies :

$$
r_{;}=v_{i:}=r_{: i}=\cdots=0 .
$$

This result could have also been obtained from the $O\left(\zeta^{p}(\Delta \lambda)^{4}\right)$ terms in the expansion of (37), .

The remaining terms in the expansion for $c$, i.e. $v_{\varepsilon}, v_{i \xi}, v_{i \varepsilon}, v_{b \varepsilon}, v_{\varepsilon \varepsilon}$ etc.. are given from equations of the type $\left(\delta_{. u u}^{\mathrm{c}} v_{k}+\overline{\mathscr{E}}_{. u w}^{\mathrm{c}} \bar{i}\right) \delta v=0$ can all be determined uniquely since the operator operating on the unknown function is always $\mathscr{F}_{\text {uu }}^{\mathrm{s}}$ which is invertible in view of (4). Upon substitution of the thus found $v\left(\zeta_{i}, \Delta \lambda_{,}, \zeta, \varepsilon\right)$ into the remaining equilibrium equation $(37)_{2}$ and after using (30). (2). (7). (8), (34), (39) one obtains the following $m$ equations between $\xi . \Delta i . \zeta$ and $\varepsilon$ :

$$
\begin{aligned}
& \varepsilon_{i} \bar{\delta}_{i k}+\frac{1}{2}\left[\sum_{i=1}^{m} \sum_{k=1}^{m} \xi_{i} \xi_{k} \delta_{i, k}+2 \Delta \lambda \sum_{i=1}^{m} \xi_{,} \delta_{i j \lambda}+2 \zeta \sum_{i=1}^{m} \xi_{i} \hat{\delta}_{i, k}+\cdots\right] \\
& +\frac{1}{6}\left[\sum_{-=1}^{m} \sum_{k=1}^{m} \sum_{1=1}^{m} \xi_{1} \xi_{k} \xi_{1} \delta_{1, k 1}+\cdots\right]+\cdots=0 \\
& \bar{E}_{i r} \equiv\left(\bar{S}_{\cdot u w}^{c} \bar{w}^{\prime}\right) u \text {. }
\end{aligned}
$$

The coefficients $\delta_{1, k} . \delta_{1, \lambda}$ and $\delta_{i, k l}$ appearing in (40)) are the same as those defined in the corresponding equilibrium equation for the perfect cuse [see eyn (10)] while $\hat{\delta}_{i, 6}$ hats been defined in (36). As expected, for $\zeta=\varepsilon=0$ the equilibrium equation for the imperfect structure with the nearly simultaneous eigenmodes $(40)$ reduces to its perfect structure counterpart (10).

The solution for the $m$ equations in (40) for the $m+3$ unknowns $\xi_{1}, \Delta \lambda, \zeta, \varepsilon$ can be expressed as a function of three parameters $\xi, \wedge$ and $Z$. This parametrization is by no means unique, but it turns out to be convenient. In analogy to the perfect case, two calses will be distinguished: first the asymmetric case, for which $\mathscr{E}_{i, k} \neq 0$ at least for some triplet of indices $(i, j, k)$. In this case the adopled parametrization is:

$$
\begin{aligned}
& \xi_{i}(\xi, \Delta \lambda, \zeta)=\bar{x}_{i}^{\prime}(\Lambda, Z) \zeta+\bar{\alpha}_{i}^{2}(\Lambda, Z) \frac{\xi^{2}}{2}+\cdots \\
& c(\xi, \Delta \lambda, \zeta)=\xi^{2}\left[\bar{\varepsilon}_{0}(\Lambda, Z)+\bar{\varepsilon}_{1}(\Lambda, Z) \xi+\cdots\right]
\end{aligned} \quad \text { where: } \Delta \lambda \equiv \xi \Lambda, \quad \zeta \equiv \xi Z, \quad \bar{\zeta}^{2} \equiv \sum_{i=1}^{m}\left(\xi_{i}\right)^{2}
$$

By inserting (41) into (40) and subsequently collecting all powers of the like orders in $\xi$, one obtains from the lowest order nontrivial term in this expansion:

$$
\bar{E}_{0} \vec{E}_{t k}+\frac{1}{2} \sum_{j=1}^{m} \sum_{k=1}^{m} \bar{\alpha}_{j}^{\prime} \bar{\alpha}_{k}^{\prime} \mathscr{E}_{i j k}+\Lambda \sum_{j=1}^{m} \bar{\alpha}_{j}^{\prime} \mathscr{E}_{i j \lambda}+Z \sum_{j=1}^{m} \bar{\alpha}_{i}^{\prime} \dot{E}_{i j}=0, \quad \sum_{i=1}^{m}\left(\bar{\alpha}_{1}^{\prime}\right)^{2}=1
$$

For fixed values of $\Lambda . Z$ the above system of $m+1$ equations for the $m+1$ unknowns $\bar{\alpha}_{i}^{-1}, \bar{\varepsilon}_{0}$ has at most $2^{m}$ real solutions. A tedious but straightforward calculation shows that the unique continuation of each solution found in (42) for the $O\left(\xi^{3}\right)$ and higher terms in (40) is assured when: 


$$
\operatorname{Det}\left[\bar{B}_{i,}\right] \neq 0, \quad \bar{B}_{i,}(\Lambda, Z) \equiv \sum_{k=1}^{m} \bar{x}_{k}^{1} E_{i ; k}+\Lambda E_{i, i}+Z \hat{E}_{i, j}
$$

Of all the possible solutions of (42) there is one of particular interest. It is the equilibrium branch that goes through the initial unloaded configuration $u=0, \lambda=0$. This solution is the unique and stable equilibrium solution that the system is going to follow during the early stages of the loading process. For a nontrivial imperfection, i.e. when $\left\|\bar{E}_{i e}\right\| \neq 0$ this solution corresponds to large negaltive values of $\Lambda$ (recall that $\Lambda=\left(\lambda-\lambda_{c}\right), \xi$ and that $\xi$ is a small positive parameter). It is not difficult to see that for $\Lambda \rightarrow-x$ the only real solution to $(42)$ is

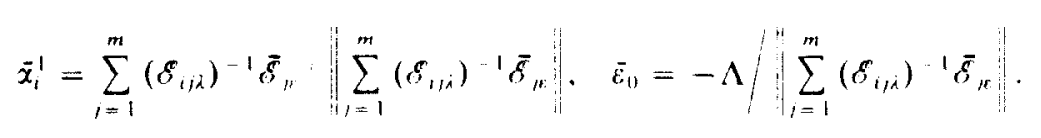

The second case to be investigated is the symmetric one. for which $\delta_{1, k}=0$ for all triplets of indices $(i . j . k)$. The adopted parametrization for the unknowns $\xi_{i} . \Delta \lambda . \zeta . \varepsilon$ in terms of the parameters $\xi . \Lambda . Z$ is :

$$
\begin{aligned}
& \xi_{i}(\xi, \Delta \lambda, \zeta)=\bar{x}_{1}^{\prime}(\Lambda, Z) \xi+\bar{x}_{i}^{2}(\Lambda, Z)_{2}^{\xi 2}+\cdots \\
& \varepsilon(\xi, \Delta \lambda, \zeta)=\xi_{2}^{\xi}\left[\bar{s}_{10}(\Lambda, Z)+\bar{\Sigma}_{1}(\Lambda, Z) \xi+\cdots\right] \\
& \text { where: } \Delta i \equiv \frac{\xi^{2}}{2} \Lambda . \quad \zeta \equiv \frac{\xi^{2}}{2} Z, \quad \xi^{2} \equiv \sum_{i=1}^{m}\left(\zeta_{i}\right)^{2} \text {. }
\end{aligned}
$$

By inserting (44) into (40) and subsequently collecting all powers of the like orders in 5 . one obtains from the lowest order nontrivial term in this expansion:

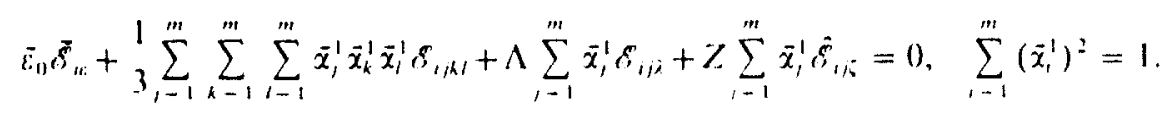

For fixed values of $\Lambda, Z$ the above system of $m+1$ equations for the $m+1$ unknowns $\bar{x}_{1}^{1}, \bar{\varepsilon}_{0}$ has at most $3^{\prime \prime \prime}$ real solutions. $\Lambda$ straightforward calculation shows that the unique continuation of each solution found in (45) for the $O\left(5^{-4}\right)$ and higher terms in (40) is assured when:

$$
\operatorname{Det}\left[\bar{B}_{i,}\right] \neq 0, \quad \bar{B}_{1,}(\Lambda, Z) \equiv \sum_{k=1}^{m} \sum_{i=1}^{m} \bar{x}_{k}^{1} \bar{x}_{1}^{1} \delta_{1, k i}+\Lambda \delta_{1, i}+Z \hat{E}_{1, k}
$$

Of all the possible solutions of (45) there is one of particular interest. It is the equilibrium branch that goes through the initial unstressed configuration $u=0, \lambda=0$. This solution is the unique and stable equilibrium solution that the system is going to follow during the early stages of the loading process. For a nontrivial imperfection this solution is identical to the one found for the asymmetric structure. This result is hardly surprising. given that the symmetry or not of the bifurcation point refers to properties of the structure near the critical load and does not influence the behavior of the structure near the zero load.

To complete the study of the imperfect structure with the nearly simultaneous buckling eigenmodes one has also to investigate the stability of the equilibrium paths. Of all the equilibrium paths found near criticality, only the one that passes through the initial unloaded state $u=0, \lambda=0$ will be investigated, since it is the actual path that the imperfect structure is going to follow during the loading process. It will be shown that the actual equilibrium path is unique and stable near $i=0$ (or equivalently as $\Lambda \rightarrow-\infty$ ). As the loading progresses, i.e. as $\lambda$ increases away from zero (or equivalently as $\Lambda$ increases away from $-\infty)$, there will be a load $\lambda_{5}$, which is called the snap-through load, for which the equilibrium branch in question will lose its stability, usually due to the attaining of a local load 
maximum. The value of $\lambda_{3}$, which obviously depends on $\zeta, \varepsilon$ and $\bar{w}$ is very significant for the design of structures that exhibit multiple eigenvalues at criticality, for it represents the maximum load attained by the actual structure before becoming unstable.

As for the perfect structure, the stability of an equilibrium solution will depend on the sign of the minimum eigenvalue $\bar{\beta}^{\text {min }}(\xi, \Lambda, Z)$ of the corresponding stability operator $\overline{\mathscr{E}}_{\text {.un }}(u, \lambda, \zeta, \varepsilon \bar{w})$. In analogy to (20), the definitions for each one of the $m$ lowest eigenvalues $\bar{\beta}(\xi, \Lambda, Z)$ of $\bar{E}_{. u m}$ and their corresponding normalized eigenvectors $\tilde{x}(\xi, \Lambda . Z)$ are :

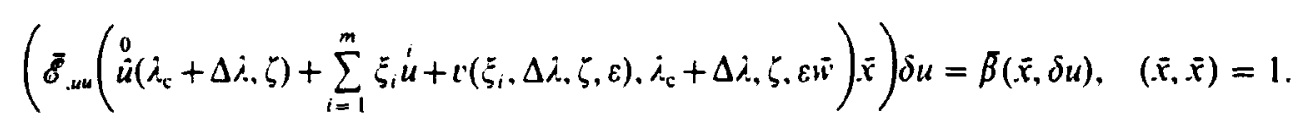

In the above definition, $\Delta \dot{\lambda}(\xi, \Lambda), \zeta(\xi, Z), \xi(\xi, \Lambda, Z), \varepsilon(\xi, \Lambda, Z)$ are given in (41) or (44) for the asymmetric or the symmetric case respectively. Note also that $v(\xi,(\xi, \Lambda, Z), \Delta \dot{\lambda}(\xi, \Lambda), \zeta(\xi, Z), \varepsilon(\xi, \Lambda, Z))$ will be given from (38). Assuming adequate smoothness with respect to $\xi$, the Taylor series expansions for $\bar{\beta}(\xi, \Lambda, Z)$ and $\vec{x}(\xi, \Lambda, Z)$ are:

$$
\begin{aligned}
& \beta(\xi, \Lambda, Z)=\bar{\beta}_{1}(\Lambda, Z) \xi+\bar{\beta}_{2}(\Lambda, Z) \frac{\xi^{2}}{2}+\cdots \\
& \bar{x}(\xi, \Lambda, Z)=\bar{x}_{0}(\Lambda, Z)+\bar{x}_{1}(\Lambda, Z) \xi+\bar{x}_{2}(\Lambda, Z) \frac{\xi^{2}}{2}+\cdots
\end{aligned}
$$

By introducing (38) into (37) and recalling (2), (7)-(8), (30), (38)-(39), one obtains (for fixed values of $\Lambda, Z$ ) by expanding about $\xi=0$ and collecting the terms of the like order in $\xi$, the following results: The $O(1)$ term yields:

$$
\bar{x}_{1}=\sum_{i=1}^{m} \bar{\chi}_{i} u
$$

Continuing with the $O(\xi)$ term in the expansion of (47) one has:

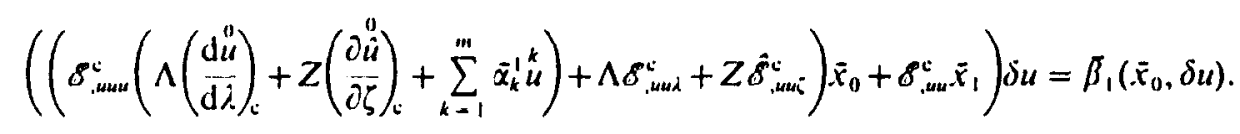

Taking $\delta u=i$ and recalling (2), and (49), (50) yields:

$$
\sum_{i=1}^{m} \bar{B}_{i}, \bar{\chi}_{i}=\bar{\beta}_{i} \bar{\chi}_{1}
$$

In the above equation the $m \times m$ matrix $\bar{B}_{1}$, is given by (43) and for the asymmetric bifurcation case, this matrix is a nonsingular one which ensures (in conjunction with the symmetry of this matrix) that all the eigenvalues $\beta_{1}$ are real and nonzero. Consequently, if $\bar{\beta}_{1}^{\min }(\Lambda, Z)$ is the minimum eigenvalue of $\bar{B}_{i j}$, then the required lowest eigenvalue $\bar{\beta}^{\text {min }}(\xi, \Lambda, Z)$ of the stability operator $\vec{B}_{\text {.uu }}$ is given by:

$$
\beta^{\text {min }}(\xi, \Lambda, Z)=\xi \beta_{1}^{\text {min }}(\Lambda, Z)+O\left(\xi^{2}\right), \quad \xi>0 .
$$

The choice of $\xi>0$ is in agreement with the assumption that $\Lambda \rightarrow-\infty$ as $\lambda \rightarrow 0$. Moreover, for the actual equilibrium path one can easily show that it is stable as $\Lambda \rightarrow-\infty$. Indeed, in view of the boundedness of $\tilde{\alpha}_{i}^{\prime}(\Lambda, Z)$ and the assumption that $\xi_{i j i}$ is a negative 
definite matrix, as $\Lambda \rightarrow-\infty$ then $\bar{B}_{i,} \rightarrow \Lambda \mathscr{E}_{1, i}$. which is a positive definite matrix. As the loading progresses (as $\lambda$ increases away from zero), it reaches a value. say $\lambda_{2}=\lambda_{2}+\Delta \lambda_{1}$. for which the actual equilibrium path becomes unstable. i.e. $\bar{\beta}^{\text {min }}(\xi, \Lambda, Z)=0$. For a fixed value of $Z$. the following relations hold between the load drop corresponding to the first instability of the actual equilibrium path $\Delta \lambda,(\xi, Z)$ in the case of an asymmetric bifurcation [see also (41)]:

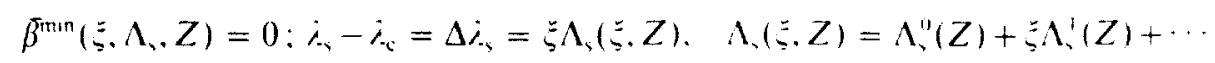

Thus. inview of (51). (52), (53). $\Lambda_{\square}^{\prime \prime}(Z)$ is the first value of $\Lambda$ as it increases from $-x$ (expected to be negative. since only structures with $\Delta \lambda_{i}<0$ are of interest) at which $\bar{B}_{1},(A, Z)$ loses its positive definiteness, i.e.

$$
\sum_{j=1}^{m} \bar{B}_{\imath \prime}\left(\Lambda_{i}^{\prime \prime}(Z), Z\right) \bar{\chi}_{i}\left(\Lambda_{i}^{\prime \prime}(Z), Z\right)=0
$$

For any other vector not colinear with $\bar{\chi}_{1}$ say $N_{1}$, one has:

$$
\sum_{i=1}^{m} \sum_{i=1}^{m} N_{i} \bar{B}_{i j} N_{1}>0
$$

The above condition, in conjunction with (53) gives us the lowest load at which the first instability in the actual equilibrium solution of the asymmetric imperfect structure will

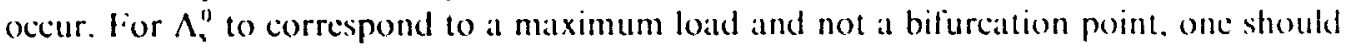
satisly:

$$
\sum_{1=1}^{m} \sum_{1=1}^{m} \delta_{1, i} \bar{x}_{,}^{\prime}\left(\Lambda_{1}^{\prime \prime}, Z\right) \bar{\chi}_{1}\left(\Lambda_{1}^{\prime \prime}, Z\right) \neq 0
$$

For the symmetric catse, the $O\left(5^{2}\right)$ torm in the expansion of $(47)$ gives $\bar{\beta}_{1}=0$ while the $O\left(5^{\prime}\right)$ term, after some lengthy but straightforward manipulations, gives:

$$
\beta^{\operatorname{mon}}(\xi, \Lambda, Z)=\frac{\xi^{2}}{2} \bar{\beta}_{2}^{\text {minn' }}(\Lambda, Z)+O\left(\xi^{\prime \prime}\right)
$$

where $\bar{\beta}_{?}^{\text {man }}$ is the lowest eigenvalue of the matrix $\vec{B}_{1}$, which is given in this case by (46). The first instability load of the actual equilibrium solution satisfies:

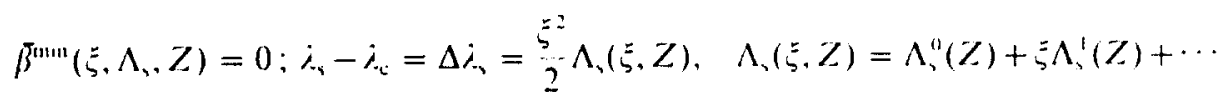

Note that $\Lambda_{4}^{\prime \prime}(Z)$ is also given in this case by (54) and satisfies $(55),(56)$ but with $\bar{B}_{11}$ taken from the definition in (46).

An alternative parametrization of the equilibrium equations of the imperfect structure in (40) which has an attractive physical interpretation can also be considered. For the asymmetric case it is :

$$
\varepsilon \equiv \eta^{2}, \quad \Delta \dot{\lambda} \equiv \eta \Lambda . \quad \zeta \equiv \eta Z . \quad \zeta_{i}(\eta, \Delta \lambda, \zeta)=\bar{x}_{1}^{\prime}(\Lambda, Z) \eta+\bar{x}_{1}^{2}(\Lambda, Z)_{2}^{\prime \eta^{2}}+\cdots
$$

while for the symmetric case it takes the form: 


$$
\varepsilon \equiv \frac{\eta^{3}}{2} . \quad \Delta \lambda \equiv \frac{\eta^{2}}{2} \Lambda . \quad \zeta \equiv \frac{\eta^{2}}{2} Z . \quad \zeta_{1}(\eta, \Delta \lambda, \zeta)=\bar{x}_{1}^{1}(\Lambda, Z) \eta+\bar{x}_{1}^{2}(\Lambda . Z)_{2}^{\eta^{2}}+\cdots
$$

This parametrization is equivalent to controlling the size of the imperfection $\varepsilon$ (or equivalently $\eta$ ) instead of controlling the amplitude $\bar{\xi}$ of the projection of the solution on the null space. The equilibrium and stability results for this new parametrization can be obtained from the equations given in this section if one sets $\tilde{\varepsilon}_{0}=1$ and drops the constraint $\sum_{i=1}^{m}\left(\bar{x}_{i}^{-1}\right)^{2}=1$. The advantage of this second parametrization is that by varying only $\Lambda$. while keeping all the other parameters fixed. one can follow the actual equilibrium path of a given imperfect structure. The advantage of the first parametrization is that it includes the perfect structure. in addition to providing a simpler solution to the corresponding worst imperfection shape problem, as it will be seen in the next section.

Finally a remark of practical importance is in order. In many applications, the functions $\hat{i}(\zeta)$ are not known explicitly, thus posing problems to the calculation of the matrix $\bar{\delta}_{1,5}[\mathrm{sec}(36)]$. For these cases, the following definition of the mode separation parameter $\zeta$ gives an approximation of the required derivative of ${ }^{i}(\zeta)$ at the critical load within an $O(\zeta)$ crror:

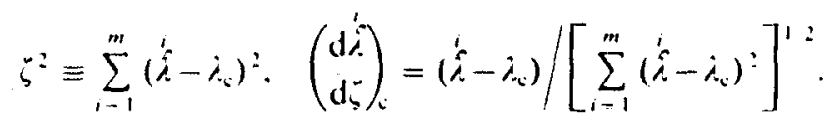

\section{WORST GEOMITRIC IMPIERTICTION SHAPE OF THIE STRUCTURE:}

The results obtained in the previous section provide the load drop $\Delta \lambda$, corresponding to the lirst load maximum encountered during a monotonic (with respect to the load parameter i.) loading of a given imperfect structure. In practical applications pertaining to the design of such structures, the control of the geometric imperlection amplitude $x$ is often casier that the control of the imperfiction shape $\bar{n}$. It is thus very important to ielentify the worst geometric imperfection shape. i.c. the shape that maximiaes $\left|\Delta \lambda_{n}\right|$.

For small values of $\xi$, the worst geometric imperfection shape is found by minimixing $\Lambda_{.}^{\prime \prime}$ (since one is interested only for $\Lambda_{, "}^{\prime \prime}<0$ ) which saltisfies (54) $(55)$, over all unit vectors $\bar{x}_{1}^{\prime}$. Consideration of all possible unit vectors $\bar{x}_{1}^{\prime}$ covers all possible equilibrium paths, als seen from (42). (45). In order to facilitate the subsequent algebra, it will be further atsumed in this section that the inner product choice in $U$ corresponds to $\delta_{1, i}=-\delta_{i,}$. Such an inner product can be catsily found in most of the applications of interest and this extrat assumption does not impair the generality of the analysis.

Two alternative formulations of the worst imperfection problem will be considered. In both the solution to the equilibrium equations (42) or (45) as well ats the limiting stability condition (54) is sought for which $\Lambda=\Lambda_{,}^{\prime}(Z)$ is minimized. However different constraints are used. In the first case (Formulation $A$ ), the amplitude $\xi$ of the projection of $u-" a(j)$ onto the null space. $V^{\prime}$ is held fixed (by enforcing the constraint $\left\|\bar{x}_{1}^{\prime}\right\|=1$ ), whereats the magnitude of the imperfection $\bar{s}_{0}\left\|\bar{E}_{u a}\right\|$ is allowed to vary. For the second case (Formulation B) the opposite is done: the amplitude $\xi$ of the projection of $u-\|(j)$ onto the null space .1 is allowed to vary (by relaxing the constraint $\left\|\bar{x}_{i}^{\prime}\right\|=1$ ), whereats the matgnitude of the imperfection is fixed an $\bar{b}_{0}=\left\|\tilde{F}_{t r}\right\|=1$.

\subsection{Formulation A}

Since from (54) (55). $\Lambda_{0}^{\prime \prime}(Z)$ is the minimum eigenvalue of $\sum_{k-1}^{m} \delta_{1, k} \bar{x}_{k}^{\prime}+Z \bar{\delta}_{1:}$ for the asymmetric case of $\Sigma_{k=1}^{\prime \prime \prime} \sum_{i=1}^{m t} \delta_{i, k i} \bar{x}_{k}^{-1} \bar{x}_{i}^{1}+Z \hat{\delta}_{i, k}$ for the symmetric case, it follows that wanted minimum of $\Lambda_{,}^{\prime \prime}(Z)$ is given by: 


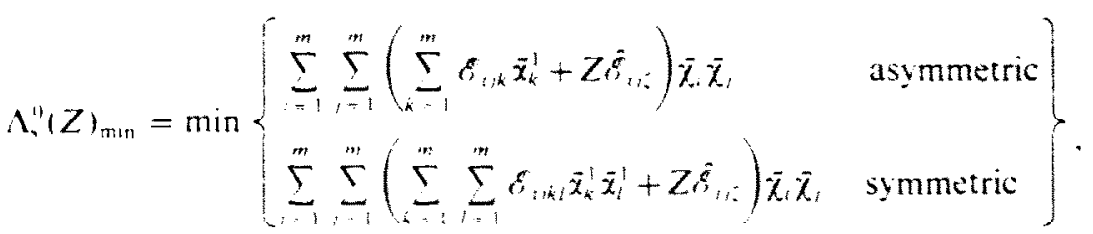

$$
\begin{aligned}
& \forall \bar{\chi}_{1} \mid=\bar{x}_{1}^{1}=1 \text {. }
\end{aligned}
$$

Since the functions to be minimized are continuous functions of their arguments and the minimization takes place over a compact set (the $m$-dimensional unit sphere), the minimum is attained for vectors $\bar{x}_{1}^{-1}$ and $\bar{\chi}_{1}$ which saltisfy the following system of $2(m+1)$ equations for the $2(m+1)$ unknowns $\bar{x}_{t}^{\prime} \cdot \bar{x}_{1} \cdot \Lambda_{1}^{\prime \prime}, M$ :

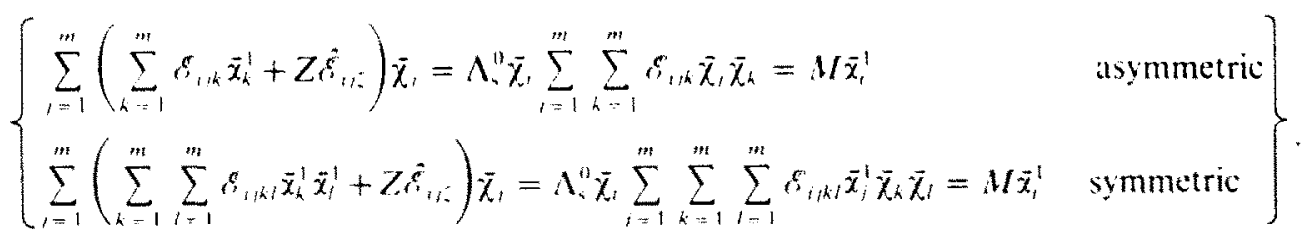

After linding the minimum solution of $(6.3)$, the corresponding worst imperfection shape $\bar{s}_{n}$ can he found from the equilibrium cquations (42) or (45) for the asymmetric or the symmetric case respectively. If the solution of $(6.3)$ also satisfies $(56)$ the worst imperfection shape corresponds to a load maximum.

Of interest is the calse of $Z=0$, for which the solution of (62) is simplified considerable. Indeed from (62) one hals

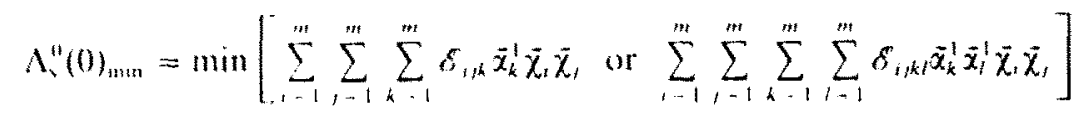

over all unit vecturs $\bar{x}_{1}^{-1}, \bar{\chi}_{1}$. It can be casily shown that the minimum exists and is athieved for $\bar{\chi}_{1}^{1}=\bar{\chi}_{1}$. Under this condition, by comparing (63) to (31), (33), one condudes that the worst imperfection shape is the one for which $\vec{f}_{k,} \times\left(x_{k}^{1}\right)_{\text {m.1. }}$, where $\left(x_{k}^{1}\right)_{\text {mmt }}$ is the direction that minimises $i_{1}$ or $i_{2}$ in the perfect structure. The required minimum $\Lambda^{\prime \prime}(0)$ is equal to the minimum possible solution $2 \lambda_{1}$ of $(31)$ for the asymmetric catse or to the minimum possible solution $3 \lambda_{2}$ of (33) for the symmetric one. In addition. $(56)$ is automatically saltisfied, thus ensuring that for $Z=0$ the worst imperfection corresponds to a maximum load. The result for the worst imperlection when $Z=0$ has been given by Ho (1974), using a different approach and invoking some additional properties for the coefficients $E_{i, k}, E_{i, k i}$ and also subsequently by Koiter (1976), who used a more elegant approach. The arguments given in support of their result do not involve the stability analysis of the imperfect equilibrium branch presented in the previous section.

Notice that the polynomial system of equations in (63), whose solution is required for the determination of the worst imperfection shape, in general hats a number of solutions that increases exponentially with $m$. Exhaustive searches to lind the wanted solution are numerically leasible for relatively low numbers $m$ of interacting modes. Fortunately, by minimizing separately each one of the two terms of the sum appearing in (62), and with the help of (36) one can deduce the following lower bound for $\Lambda^{\prime \prime}(Z)_{\min }$ :

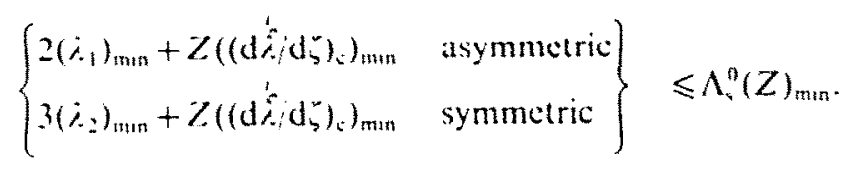

\subsection{Formulation $B$}

The results in (62)-(63) have been obtained for the case of a fixed projection amplitude parameter $\xi$. It is interesting to ask the same question about the worst imperfection for the 
case where the amplitude of the imperfection is fixed, i.e. $\bar{\varepsilon}_{0}=1$ [see (59)-(60)]. For this case one seeks to minimize $\Lambda_{?}^{0}(Z)$ satisfying the equilibrium conditions (42) or (45) as well as the conditions for a limit point (54)-(56) under the constraint $\left\|\vec{E}_{i s}\right\|=1$ instead of the constraint $\left\|\bar{x}_{1}^{1}\right\|=1$. Hence, the extremality condition that has to be satisfied by $\Lambda_{\mathbf{s}}^{0}(Z)$ is:

$$
\frac{\partial \Lambda_{\mathrm{s}}^{0}}{\partial \bar{E}_{i \varepsilon}}+\psi \bar{E}_{i s}=0
$$

where $\psi$ is a scalar Lagrange multiplier.

For either the asymmetric or the symmetric case, differentiation of (42) or (45) with respect to $\bar{\delta}_{\text {is }}$. multiplication of $\bar{\chi}_{i}$ and summation with respect to the index $i$ gives in view of (54):

$$
\overline{\chi_{1}}+\left(\sum_{i=1}^{m} \sum_{j=1}^{m} \mathscr{E}_{i j \lambda} \bar{x}_{j}^{1} \bar{\chi}_{i}\right) \frac{\partial \Lambda_{s}^{0}}{\partial \bar{E}_{l s}}=0
$$

Since only load extrema are of interest, (56) is satisfied. From (65) and (66) one concludes that at extremality $\bar{E}_{i k} \propto \bar{\chi}_{i}$ and consequently from (42) or (45) and (54) the following condition has to be satisfied at the minimum $\Lambda_{3}^{0}(Z)$ :

$$
\sum_{j=1}^{m} \sum_{k=1}^{m} \bar{B}_{i}\left(\bar{B}_{1 k}+\phi\left(\Lambda_{i}^{0} \bar{\delta}_{j k \lambda}+Z \hat{\delta}_{j k i}\right)\right) \bar{\alpha}_{k}^{1}=0
$$

where $\phi=1$ for the asymmetric case while $\phi=2$ for the symmetric one.

To find the required worst geometric imperfection shape, i.e. to find $\bar{\alpha}_{1}^{-1}, \Lambda_{1}^{0}$, one has to solve the system of $m+1$ equations (67) complemented by the constraint

$$
\left\|\sum_{k=1}^{m}\left(\bar{B}_{1 k}+\phi\left(\Lambda_{i}^{0} \tilde{E}_{1 k \lambda}+Z \hat{E}_{1 k j}\right)\right) \bar{x}_{k}^{-1}\right\|=\phi+1
$$

Notice however that the polynomial system in (67) has a rather high number of possible nontrivial real solutions which increases exponentially with the number of interacting modes $m$. Exhatustive searches to find the required solution are numerically feasible for relatively low numbers, $m$, of interacting modes.

Once again, of interest is the solution of the worst imperfection shape problem for $Z=0$. It will be shown that for this case, the problem is once more reduced to the solution of (12) or (14) and the required minimum $\Lambda_{s}^{0}(0)$ is equal to the minimum possible solution $2 \lambda_{1}$ of $(12)$ for the asymmetric case or to the minimum possible solution $3 \lambda_{2}$ of (14) for the symmetric one.

Indeed, for $Z=0$ and for the inner product choice that gives $\mathscr{E}_{i j d}=-\delta_{i j}$ one can rewrite the extremality condition (67) as:

$$
\sum_{j=1}^{m} \sum_{k=1}^{m}\left(\bar{B}_{i j}+\phi \Lambda_{i}^{0} \bar{\delta}_{i j k}\right) \bar{B}_{j k} \bar{\alpha}_{k}^{1}=0
$$

The wanted result $\sum_{k-1}^{m} \bar{B}_{j k} \bar{\alpha}_{k}^{-1}=0$ follows by establishing that the factor in parenthesis in (68) is a positive definite matrix. For this purpose, note that $\bar{B}_{i,}$ is positive semidefinite by (54-55) and that the second term in the parenthesis is a positive multiple of the identity. Hence the lowest eigenvalue of the factor in parenthesis is $-\phi \Lambda_{\mathrm{s}}^{0}$ which is positive.

Notice that when $Z=0$, the worst imperfection shape problem has the same answer, irrespective on whether $\xi$ or $\varepsilon$ is controlled. For $Z \neq 0$, one expects in general different solutions to (63) or (67). To find the minimum $\Lambda_{s}^{0}(Z)$ for $Z \neq 0$ and the corresponding worst imperfection shape, an incremental Newton-Raphson method based on (63) or (67) can be used, starting with the perfect structure solution obtained for $Z=0$, and tracking 
the solution for increasing values of $|Z|$. This procedure is computationally more appealing than finding directly all solutions of (63) or (67). However, there may be some value of $Z$ beyond which this incremental procedure no longer produces an absolute minimum for A.".

\section{CONCLUDING REMARKS}

So far we have established a general theory for the equilibrium and stability of arbitrary (continuum or discrete) imperfect elastic structures with nearly simultaneous eigenmodes. From the stability analysis one can subsequently determine the worst possible shape of the geometric imperfection that will minimize the first local maximum load of the structure. The analysis presented is asymptotic in nature and the fundamental question pertains to the range of validity of the employed asymptotic expansions. It has long been known. especially in the shells literature [see for example the review article by Hutchinson and Koiter (1970)]. that the range of validity of the asymptotic expansions used in many applications is restricted. Such results are often based on first term asymptotic analyses where only a few modes are allowed to interact at the time, usually for reasons of analytical tractability. Had it been possible for all the nearly simultaneous modes to be taken into account. in addition to the inclusion of the higher order terms, one can conjecture that the conclusions about the validity of these expansions might improve. The so-called multimode methods used in the literature are also an improvement upon the first order asymptotics, if all the relevant interacting modes are included. These methods can be combined with the results presented here as an alternative to the inclusion of the higher order terms in the alsymptotic expansions.

At iny rate, the solution of the worst imperfection shape problem proposed here, will have to be considered in conjunction with the solution of the full set of nonlinear equilibrium equations of the structure in question. The results of this analysis are in a way complementary to the full (usually numerical) solution of the imperfect structure for they provide with an intelligent selection of the imperfection shape that will produce the maximum load drop $\Delta i$, in the reil structure. This methodology establishes a deterministic alternative to the statistical methods proposed for the cylindrical shell case by Arbocz (1987). A combination of both methods might also be useful. It is even conceivable that the worst imperfiction shape could be used to obtain estimates or bounds to the probability of failure for random imperfections.

One can think of applications where the number of interacting modes $m$ to be considered is high. Since the determination of the worst imperfection shape requires the solution of a nonlinuar polynomial system with $m$ simultaneous equations, one faces the problem of computing all the roots of such a system in order to pick up the required answer. Numerical algorithms for such a lask to exist [based on homotopy methods-see for example Chow 't al. (1979) for the theoretical foundations of these methods and Morgan (1987) for the algorithmic application]. However, the required solution time might be exceedingly high fir high salues of $m$, since the number of solutions to (65) or (66) grows exponentially with m. For these ciases, one will have to rely on special properties of these equations in order to devise quicker solution procedures. For certain structures with a high number of interacting modes, one of course has the option of using an amplitude modulation type method [sec for cxample Poticr-Ferry (1987) and references quoted thercin], for which however a worst imperfection shape analysis has not been developed thus far.

Icknowledelements - Support by N.S.F. Grant MSS-910,3227 to the University of Michigan is gratefully acknowledged.

\section{REFERENCES}

Arhoc7. J (1987). Post-buckling behavior of structures. numerical techniques for more complicated structures. In Bucklina and Pest-huckling (Edited by J. Arbocz, M. Potier-Ferry, J. Singer and V. Tvergaard), Lecture Notes in Physics. Vol. 288. pp. 83-142. Springer. Berlin. 
Budiansky. B. (1974). Theory of buckling and postbuckling behavior of elastic structures. In .Adtances in .Applied Mechanics (Edited by C. H. Yih), pp. 1-65. Academic Press. New York.

Byskov. E. and Hutchinson. J. W. (1977). Mode interaction in axially stiffened cylindrical shells. fl/t.t $J / 15$. 941-948.

Chow. S. N.. Mallet-Parret, J. and Yorke. J. A. (1979). A homotopy method for locating all zeroes of a system of polynomials. In Functional Differential Equations and Approximation of Fixed Prints (Edited by $\mathrm{H}$. $\mathrm{O}$. Peitgen and H. O. Walther). Lecture Notes in Mathematics. Vol. 730. pp. 228-237. Springer. Berlin.

Healet. T. J. (1988). A group-theoretic approach to computational bifurcation problems with symmetry. Comp. Meth. Appl. Mech. Engng 67, 257-295.

Ho. D. (1974). Buckling load of non-linear systems with multiple eigenvalues. Int. J. Solids Structures 10, $1315-$ 1330.

Hutchinson, J. W. and Koiter, W. T. (1970). Postbuckling theory. Appl. M/ch. Ret. 23. 1353-1366.

Iooss. G. and Joseph. D. D. (1980). Elementary Stability and Bifurcation Theory. Springer. Berlin.

Knops. R. J. and Wilkes. E. W. (1973). Theory of elastic stability. Hondhook of Physics. Vol. Vla. pp. 125-302. Springer, Berlin.

Koiter, W. T. (1945). On the stability of elastic equilibrium. Thesis. Delft University (in Dutch). English Translation: NASA Techn. Trans. F-10.833.

Koiter. W. T. (1965). The energy criterion for stability for continuous elastic bodies. Proc. Kon. Ned. Akad. Wetensch B68, 178-202.

Koiter, W. T. (1976). Current trends in the theory of buckling. Buckling of Structures. Proceedings of the IUTAM Symposium held at Cambridge MA/USA on June 17-21. 1974, pp. 178-202. Springer, Berlin.

Morgan. A. P. (1987). Computing all solutions to polynomial systems using homotopy continuation. Appl. W/ath. Compur. 24, 101-113.

Potier-Ferry. M. (1979). Perturbed bifurcation theory. J. Diff. Equat. 33, 112-146.

Potier-Ferry, M. (1987). Foundations of elastic postbuckling theory. In Buckling and Post-huckling (Edited by J. Arbecz. M. Potier-Ferry, J. Singer and V. Tvergatard). Lecture Notes in Physies. Vol. 288, pp. 83-142. Springer. Berlin.

Simpson. H. C. and Spector, S. J. (1987). On the positivity of the second variation in finite slasticily, arch. Rat. Merh. Anal. 98, 1 -30.

Thompson, J. M. T. and Hunt, G. W, (1973). A General Theory for Flastic Stitbility. John Wiley. New York. 